\title{
The Light Stop Quark with Small Stop-Neutralino Difference in the MSSM
}

\author{
C. Milsténe, M. Carena \\ FNAL, Batavia, IL 60510, USA \\ A. Freitas \\ Zurich U., 8057 Zurich, Switzerland \\ A. Finch, A. Sopczak \\ Lancaster U., Lancaster LA1 4YB, UK \\ H. Nowak \\ DESY, D-15738 Zeuthen, Germany
}

\begin{abstract}
The MSSM can explain electro-weak symmetry breaking if one scalar top quark (stop) is light. In addition, in this framework, the neutralino is a good dark matter candidate and for small stop-neutralino mass differences $D_{m}=30$ $\mathrm{GeV}$, co-annihilation plays an important role to match the results from WMAP and SDSS for the relic density in the universe. In this scenario, the stops mainly decays into charm and neutralino, making its discovery difficult at hadron colliders due to background and trigger limitations. We present results for the discovery reach of the ILC for a DM candidate as low as $0(5 \mathrm{GeV})$ based on a realistic experimental simulation. Moreover, the stop parameters could be measured with high precision.
\end{abstract}

\section{INTRODUCTION}

Template documents The Scalar top studies for small visible energy in the detector at the Linear Collider are motivated by electroweak symmetry in particle physics and by the nature of dark matter and baryogenesis in cosmology. They both suggest the existence of new symmetries within the reach of the next generation of colliders. Recently, dark matter energy density of the universe has been precisely measured by the Wilkinson Microwave Anisotropy Probe (WMAP), in combination with the Sloan Digital Survey (SDSS) [1], to be $\Omega_{\mathrm{CDM}} h^{2}=0.1126_{-0.0181}^{+0.0161}$ at the $95 \%$ C.L. Here $\Omega_{\mathrm{CDM}}$ dark matter energy density normalized to the critical density and $\mathrm{h}$ is the Hubble parameter in units of $100 \mathrm{~km} / \mathrm{s} / \mathrm{Mpc}$. The super-symmetry with $\mathrm{R}$ parity conservation provides a stable neutral dark matter candidate, which in the MSSM is the neutralino, with mass and cross-section within the electro-weak energy scale. The neutralino-stop co-annihilation region with small mass difference $\Delta m=\left(m \tilde{t}_{1}-m \tilde{X}_{1}^{0}\right)=15-30 \mathrm{GeV}$, provides a relic density compatible with the WMAP observation. Of interest is the decay of the stop pair produced into 2 soft charm jets and missing energy. The study involves a detailed experimental analysis with a realistic detector simulation including a CCD vertex detector for flavor tagging, which may be a possible benchmark for vertex detector and Forward Calorimeter. The Dark-Matter study is further developed in a presentation in the Cosmology session of this conference, by A. Freitas. This has been published in [2] and updates a presentation made at the 2005 International Linear Collider Workshop Stanford [3]. More realistic systematic errors are taken into account since [3] and one can show that the discovery reach of the light stop can be attained at the ILC with a luminosity as low as $10 \mathrm{fb}^{-1}$

\section{EXPERIMENTAL SIMULATIONS}

This study is centered on the light stop with a small mass difference with the lightest neutralino. It predominantly decays into a charm quark jet and the lightest neutralino. The signature for a stop pair production in an $e^{+} e^{-}$ machine is two soft charm jets and missing energy. The separation of soft jets from the background is challenging since the background cross-sections are often several orders of magnitudes higher than the signal, see Table I. This study is performed for a luminosity of $500 \mathrm{fb}^{-1}$ and at a center of mass energy of $500 \mathrm{GeV}$. The signal and background

\section{ALCPG0317}


Table I: Cross-sections for $\mathrm{ECM}=500 \mathrm{GeV}, \cos \tilde{\Theta} t=0.5$

\begin{tabular}{|c|c|c|c|c|}
\hline \multicolumn{2}{|c|}{ Process } & \multicolumn{3}{|c|}{ Cross-section [pb] } \\
\hline \multicolumn{2}{|c|}{$P\left(e^{-}\right) / P\left(e^{+}\right)$} & $0 / 0$ & $-80 \% /+60 \%$ & $-60 \%$ \\
\hline \multirow[t]{4}{*}{$\tilde{t}_{1} \tilde{t}_{1}^{*}$} & $m_{\tilde{t}_{1}}=120 \mathrm{GeV}$ & 0.115 & 0.153 & 0.187 \\
\hline & $m_{\tilde{t}_{1}}=140 \mathrm{GeV}$ & 0.093 & 0.124 & 0.151 \\
\hline & $m_{\tilde{t}_{1}}=180 \mathrm{GeV}$ & 0.049 & 0.065 & 0.079 \\
\hline & $m_{\tilde{t}_{1}}=220 \mathrm{GeV}$ & 0.015 & 0.021 & 0.026 \\
\hline \multicolumn{2}{|c|}{$W^{+} W^{-}$} & 8.55 & 24.54 & 0.77 \\
\hline \multicolumn{2}{|l|}{$Z Z$} & 0.49 & 1.02 & 0.44 \\
\hline \multicolumn{2}{|c|}{$W e \nu$} & 6.14 & 10.57 & 1.82 \\
\hline \multicolumn{2}{|c|}{$e e Z$} & 7.51 & 8.49 & 6.23 \\
\hline \multirow{2}{*}{\multicolumn{2}{|c|}{$\begin{array}{l}q \bar{q}, q \neq t \\
t \bar{t}\end{array}$}} & 13.14 & 25.35 & 14.85 \\
\hline & & 0.55 & 1.13 & 0.50 \\
\hline \multicolumn{2}{|c|}{ 2-photon, $p_{\mathrm{t}}>5 \mathrm{GeV}$} & 936 & & \\
\hline
\end{tabular}

events are generated with PYTHIA 6.129 [4]. using CIRCE [5] for the Beamstrahlung and with additional private code used in Ref.[6]. A realistic detector simulation based on the fast simulation SIMDET [7] includes a CCD vertex detector for flavor tagging. The charm tagging is used to separate apart the background from the signal. The crosssections of the signal and relevant background are given in Table I. They have been computed using the Monte-Carlo Ref.[8] and with GRACE 2.0 Ref.[9] and CompHEP 4.4 Ref.[10] when applicable. The cross-sections have been calculated for a mixing angle $\cos \tilde{\Theta} t=0.5$ and for 3 beam polarization combinations. The negative/positive values refer to left/right polarization respectively. To avoid infrared divergence, a cut at $p_{t}>5 \mathrm{GeV} / \mathrm{c}$ is applied for the $2 \gamma$ background.

\subsection{Pre-selection and Selection Cuts}

We perform a 2 stages set of cuts in order to separate signal from background. In the first step, the pre-selection the bulk of the background is removed to alleviate the simulation load. The cuts are listed below with the main backgrounds they suppress next to them. For most of the backgrounds, the missing momentum comes from particles lost in the beam pipe, whereas, in the signal the neutralinos are responsible for the large missing energy and large missing pt.

- $4<N($ ChargedTracks) $<50$ (BG: tt, $\mathrm{l}=\mathrm{e}, \mu, \gamma) ; p t>5 G e V / c$ (BG:2 $\gamma, \mathrm{qq})$

- $\left|\cos \Theta_{\text {Thrust }}\right|<0.8$ (BG: all backgrounds) ; $\mid$ plong $/$ ptot $\mid<0$ (BG: all)

- Evis $<0.75$ (BG: all) ; $m_{\text {inv }}<200$ (BG: all)

The selection cuts are given below with the main backgrounds they suppress.

- $N$ jets $=2(\mathrm{BG}: \mathrm{W}$ and qq $) ; E v i s<0.4 / E c m\left(\mathrm{BG}: W^{+} W^{-}, Z Z, q \bar{q}, t \bar{t}\right)$

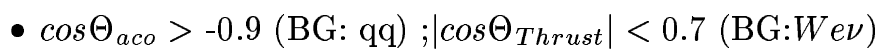

- $P t>12 \mathrm{GeV}(\mathrm{BG}: 2 \gamma) ; 60 \mathrm{GeV}<$ mjet $_{i n v}<90 \mathrm{GeV}(\mathrm{BG}: W e \nu)$

A charm-tagging implemented to reduce the main background left, $W e \nu$, is based on a neural network analysis [11]. The selection cuts are described to a greater extent with their effect in [2].and [3]. In Table II the number of signal and background events is shown after all the cuts and for a luminosity of $500 \mathrm{fb}-1$

\section{ALCPG0317}


Table II: Number of Background and Signal Events after the Selection Cuts for 500 fb-1 Luminosity

\begin{tabular}{|l|c|r|c|}
\hline Background & $\begin{array}{c}\text { Total after Selection } \\
\text { For } 500 \mathrm{fb}^{-1}\end{array}$ & Signal & $\begin{array}{c}\text { Total after Selection } \\
\text { For } 500 \mathrm{fb}^{-1}\end{array}$ \\
\hline$W^{+} W^{-}$ & 145 & $m_{\tilde{t}}=140 \mathrm{GeV} \Delta m=20$ & 9720 \\
$Z Z$ & 257 & $\Delta m=40$ & 4700 \\
$W e \nu$ & $\Delta m=80$ & 4840 \\
$e e Z$ & 5044 & $m_{\tilde{t}}=180 \mathrm{GeV} \Delta m=20$ & 6960 \\
$q \bar{q}, q \neq t$ & 36 & $\Delta m=40$ & 4925 \\
$t \bar{t}$ & 160 & $\Delta m=80$ & 3675 \\
$2 \gamma, p t>5 \mathrm{GeV}$ & 38 & $m_{\tilde{t}}=220 \mathrm{GeV} \Delta m=20$ & 2600 \\
& $<164$ & $\Delta m=40$ & 1815 \\
& & $\Delta m=80$ & 1410 \\
\hline
\end{tabular}

Table III: Signal Efficiency after final event Selection for different $m \tilde{t}_{1}$ and $\Delta \mathrm{m}$

\begin{tabular}{|l|c|c|c|c|}
\hline$\Delta \mathbf{~ m}$ & $m_{\tilde{t}_{\mathbf{1}}}=\mathbf{1 2 0 G e V}$ & $m_{\tilde{t}_{\mathbf{1}}}=\mathbf{1 4 0 G \mathbf { G e V }}$ & $m_{\tilde{t}_{\mathbf{1}}}=\mathbf{1 8 0 G e V}$ & $m_{\tilde{t}_{\mathbf{1}}}=\mathbf{2 2 0 G} \mathbf{G e V}$ \\
\hline $80 \mathrm{GeV}$ & & $10 \%$ & $15 \%$ & $19 \%$ \\
$40 \mathrm{GeV}$ & & $10 \%$ & $20 \%$ & $24 \%$ \\
$20 \mathrm{GeV}$ & $17 \%$ & $21 \%$ & $28 \%$ & $35 \%$ \\
$10 \mathrm{GeV}$ & $19 \%$ & $20 \%$ & $19 \%$ & $35 \%$ \\
$5 \mathrm{GeV}$ & $2.5 \%$ & $1.1 \%$ & $0.3 \%$ & $0.1 \%$ \\
\hline
\end{tabular}

\subsection{The discovery reach for the light stop at the ILC}

The efficiencies are calculated from the simulations with $\mathrm{Ecm}=500 \mathrm{GeV}$, assuming $100 \%$ cross-section to the process studied. The expected number of signal events is calculated for the whole parameter region of interest by interpolating the parameter points $\left(m_{\tilde{t}_{1}}, m_{\tilde{X}_{1}}^{0}\right)$ of Table IIItogether with the production cross-section given in Table I.

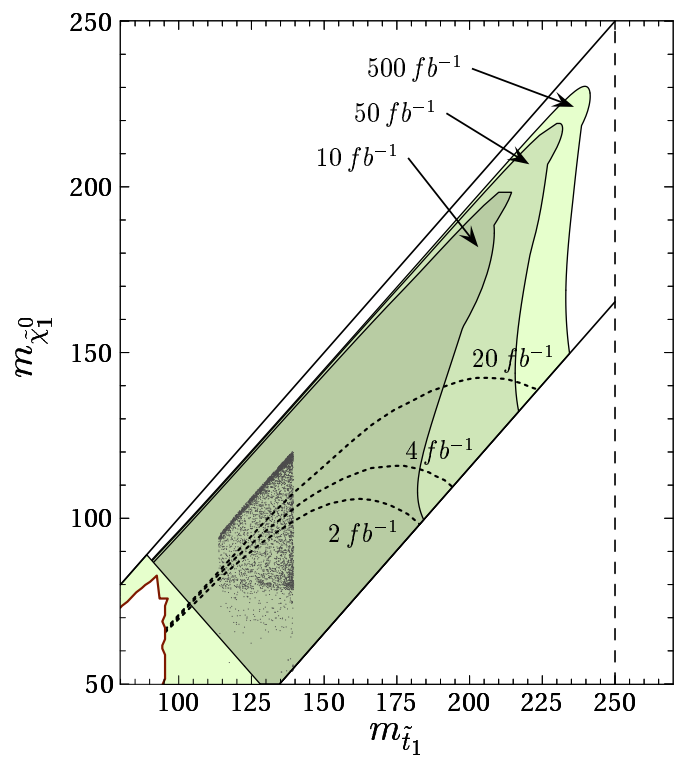

Figure 1: The Discovery reach for the light stop at the ILC 


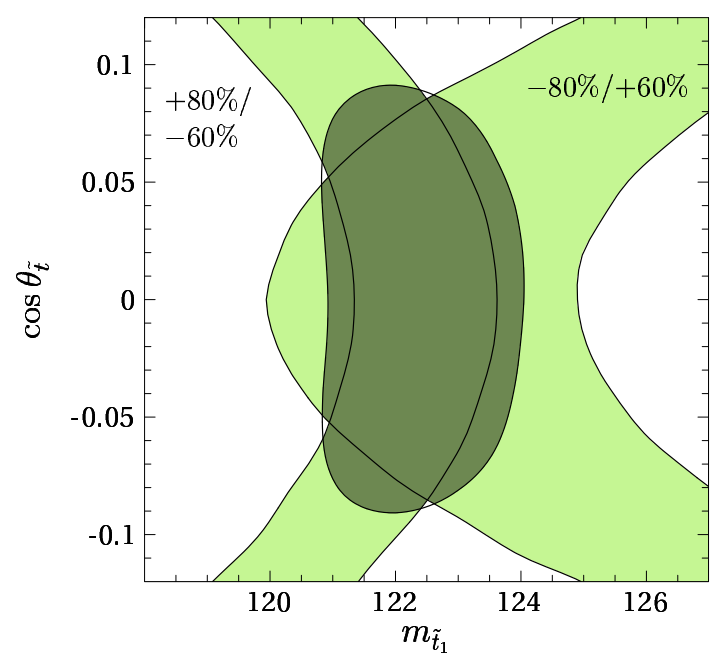

Figure 2: Light stop mass vs.mixing angle.

The shaded region in the stop-neutralino mass plane shown in Figure 1 corresponds to a $5 \sigma$ discovery, for a luminosity of $500 \mathrm{fb}^{-1}, 50 \mathrm{fb}^{-1}$ and even $10 \mathrm{fb}^{-1}$. This is shown for a significance $\mathrm{S} /(\mathrm{S}+\mathrm{B})>5$, where $\mathrm{S}=\epsilon \times \sigma \times L$ with $\epsilon$ taken from Table III and $\sigma$ from Table I as well as the background information B. The sharp cut at the bottom of the shaded region comes from heavier stops for which the 3-body decay $W b \tilde{X}_{1}^{0}$ dominates. In the left corner, the light grayish region corresponds to a stop which is so light that the top decays into stop and neutralino. Those 2 decay channels where not studied here. The dotted dark gray region is consistent with WMAP and baryogenesis. In the figure the dotted lines corresponding to the light stop reach by the Tevatron for different luminosities [12] and the white left corner to the exclusion by LEP.

\section{STOP PARAMETERS DETERMINATION}

A light stop in the neutralino co-annihilation mass range and with a small mixing angle will favor a supersymmetric dark matter hypothesis that we will check against a consistent set of supersymmetric parameters. A specific MSSM point has been chosen to be compatible with the mechanism of electro-weak baryogenesis, as in [2], $m \tilde{U}_{3}^{2}=-99^{2} \mathrm{GeV}^{2}$, $m \tilde{Q}_{3}=4200 \mathrm{GeV}, \mathrm{At}=-1050 \mathrm{GeV} ; M_{1}=112.6 \mathrm{GeV}, M_{2}=225 \mathrm{GeV},|\mu|=320 \mathrm{GeV}, \Phi_{\mu}=0.2, \tan \beta=5$ and corresponds to dark matter within the WMAP range with $\Omega_{\mathrm{CDM}} h^{2}=0.1126$. and for that point with the stop mass $m \tilde{t}_{1}=$ $122.5 \mathrm{GeV}$ and the neutralino mass $m \tilde{X}_{1}^{0}=107.2 \mathrm{GeV}$ with a small $\Delta m=15.2 \mathrm{GeV}$ and a right-chiral stop, with $\cos \tilde{\Theta} t=0.0105$. The dark matter relic density is computed with the code used in [13]. The beam polarization allows to use the measurement of the cross-section of the (stop, anti-stop) for 2 different combinations of the beam polarization. The two non-zero polarization combinations and cross-sections from Table I allowed us to extract both mass and mixing angles of the stop. The systematic errors accounted for are described in detail in [2]. They include a systematic error of the mass measurement of the neutralino of $\simeq 0.1 \mathrm{GeV}$, an error of $\simeq 0.5 \%$ of the degree of polarization measured and an error of $\simeq 5 \times 10^{-4}$ of the integrated luminosity. The theoretical uncertainty of background simulations is $\mathrm{dB} / \mathrm{B} \simeq 0.3 \%$ and stop hadronization fragmentation $\simeq 1 \%$. In Figure 2 , each band corresponds to the cross-section measurement with one of the non-zero polarization combinations. The error band width is given by the errors. The region at the overlap of the two bands is shown in Figure 2and allows to determine the stop mass and the mixing angle to be $m \tilde{t}_{1}=(122.5 \pm 1.0) \mathrm{GeV}, \cos \Theta \tilde{t}<0.074$, which for our accuracy is consistent with zero and corresponds to a $\tilde{t}$ right handed.

\section{ALCPG0317}


